\title{
DIE SPRACHE DES MARKETINGS UND IHRE ÜBERSETZUNG: MORPHOLOGISCHE UND SEMANTISCHE ASPEKTE DER TERMINOLOGIE
}

\author{
Anastasia Konovalova \\ Guadalupe Ruiz Yepes \\ anastasia.konovalova@hs-heilbronn.de \\ guadalupe.ruiz-yepes@hs-heilbronn.de \\ Hochschule Heilbronn
}

\section{Zusammenfassung}

Mit der schnellen Entwicklung der Fachsprachen im Bereich Wirtschaft wird auch die Wichtigkeit der Fachkommunikation in internationalen Unternehmen immer deutlicher. Für die Fachübersetzer wirtschaftlicher Texte bedeutet diese Entwicklung oft eine große Herausforderung. Dieser Aufsatz widmet sich der kontrastiven Analyse der Terminologie des Marketings für das Sprachenpaar Deutsch-Spanisch. Das analysierte Korpus besteht aus wirtschaftswissenschaftlichen Beiträgen aus dem Bereich Marketing, die in spanischen und deutschen Fachzeitschriften veröffentlicht wurden.

\begin{abstract}
"The language of marketing and its translation: morphological and semantic aspects of terminology"

The importance of languages for special purposes in multinational companies grows with each passing day. Simultaneously, the language of business, especially in the field of terminology, is developing at breakneck speed. In this respect, the work of translators in the field of business is becoming a real challenge. With this paper we hope to shed some light on the challenges of translating terminology in the field of marketing for the language pair German-Spanish. The genres represented in the corpus are mainly scientific papers published in specialised journals.
\end{abstract}

Schlüsselwörter: Fachsprache. Fachübersetzen. Marketing. Terminologie.

Keywords: Language for special purposes. Specialised translation. Marketing. Terminology. 
Manuscript received on June 30, 2015 and accepted for publication on October 16, 2015. 


\section{Einführung}

Das Fachübersetzen ist heute der wichtigste Arbeitsbereich professioneller Übersetzer. Schon im Jahr 1989 ergab eine Studie mit einer Umfrage von Schmitt (1990: 97f), dass der weitaus größte Teil der in der Praxis zu übersetzenden Texte technischer Natur waren. $76 \%$ der befragten Übersetzer gaben an, in ihrem Alltag hauptsächlich mit technischen Texten zu tun zu haben. Dahinter folgten die Bereiche Wirtschaft (12\%) und Recht (6\%). Bisher ist diese Tatsache von keiner anderen Studie widerlegt worden, weshalb wir davon ausgehen können, dass sich diese Verhältnisse kaum verändert haben. Dieser Aufsatz beschäftigt sich mit dem Übersetzen der Fachsprache des Marketings als Teil der Wirtschaftssprache und kann Übersetzer dazu anregen, sich mit der Terminologie des Marketings auseinanderzusetzen. Der Aufsatz hat also das Ziel, die morphologischen und semantischen Eigenheiten der Marketingterminologie zu analysieren und somit das Fachübersetzen in diesem Bereich zu erleichtern.

Jeder, der sich in der Finanz- und Wirtschaftswelt auskennt, weiß, dass die englische Sprache sehr oft als internationale Lingua Franca fungiert die steigende Globalisierung der Wirtschaft hat dazu geführt, dass viele Geschäfte und Wirtschaftstransaktionen auf Englisch ablaufen. Dementsprechend ist der Einfluss der englischen Sprache auf die Fachterminologie im Bereich Wirtschaft in anderen Sprachen sehr hoch. Die "falschen Freunde" sind genau aus diesem Grund einer der Aspekte der Fachkommunikation im Bereich Wirtschaft, der am meisten in den aus der Übersetzungswissenschaft stammenden Studien analysiert wird. Laut Pérez Berenguel (2003: 619) werden zum Beispiel in der spanischen Presse englische Wörter nicht durch ihre spanischen Äquivalente in der Wirtschaftssprache ersetzt, sondern oft durch „morphologisch verwandte Wörter“, also durch falsche Freunde („subida dramática de los tipos de interés" statt "subida importantelespectacular de los tipos de interés" für „dramatic rise of interest rates"). Dieses Phänomen darf nicht mit dem richtigen Gebrauch von Wörtern verwechselt werden, die zwar ursprünglich in der englischen Sprache im wirtschaftlichen Kontext verwendet wurden, aber schnell in der spanischen Wirtschaftssprache 
Akzeptanz gefunden haben,weil sie aus dem Lateinischen stammen (futuros für futures oder opciones für options). Eine dritte Kategorie bilden laut Pérez Berenguel (ebd.) die englischen Wörter, die zwar eine Entsprechung auf Spanisch haben, aber sich trotzdem aus sprachökonomischen Gründen in der spanischen Wirtschaftssprache durchgesetzt haben (leasing statt alquiler con opción a compra oder marketing statt comercialización). Diese letzte Kategorie lässt sich auch in der deutschen Wirtschaftssprache feststellen (Leasing statt Verpachtung/Vermietung und Marketing statt Vermarktung). Doch die Verwendung überflüssiger Anglizismen ist nur eines der vielen Phänomene, die in der Übersetzung von wirtschaftlichen Texten beobachtet werden können. Die durch Verkürzungen entstandenen Wörter stellen auch Probleme verschiedenster Art dar, zum Beispiel, wenn es darum geht, die witzige Pointe eines Wortes zu übertragen (STRIPS: Separate Trading of Registered Interest and Principal Securities) [(vgl. Alcaraz Varó 2001: 14-15) nach Gallego Hernandez 2012: 97], oder wenn eine Verkürzung mehrere Bedeutungen hat (ABS: American Bureau of shipping, Anti-Bloc System, Associate of the Building Societies Institute) [(vgl. Gómez Moreno 1995: 578) nach Gallego Hernandez 2012:97].

Diese und andere Aspekte der Terminologie werden ausführlich in verschiedenen Studien behandelt, da man lang der Meinung war, dass Fachsprachen sich nur durch ihre Fachterminologie von der allgemeinen Sprache differenzierten. Diese These ist jedoch schon lange überholt. Schon in den achtziger Jahren haben sich Forscher der Syntax von Fachsprachen gewidmet und Aspekte, wie z.B. das Vorkommen von Hypotaxen und Parataxen, in den Texten analysiert oder die Thema-Rhema-Gliederung untersucht (vgl. Gerzymisch-Arbogast 1987). Ein anderer nicht zu vernachlässigender Aspekt ist auch die Übermittlung von rhetorischen Figuren. Die Übersetzung von Metaphern in der Wirtschaftssprache, um nur ein Beispiel zu nennen, stellt ebenfalls eine Herausforderung für Übersetzer dar. Eine Studie von Serón Ordóñez (2005: 205-249) an einem parallelen Korpus für die Sprachenkombination Englisch-Spanisch ergab, dass bei über 70\% der Fälle die Übersetzer die Metaphern des Englischen auf Spanisch beibehalten hatten. Nur bei 20\% der Fälle entschieden sich die Übersetzer für eine andere Metapher, vorwiegend wenn es sich um Metaphern aus dem Bereich der Schifffahrt handelte. Die restlichen 10\% machten Metaphern aus den Bereichen Tierreich und Sport aus und wurden von den Übersetzern paraphrasiert.

Für all diese Formen des Fachübersetzens gilt aber, dass es immer mehr einem Tätigkeitskomplex ähnelt, „der zwar auch das Übersetzen im eigentlichen Sinn beinhaltet, meist aber andere Managementfähigkeiten und 
unterschiedliche Wissensbereiche miteinander kombiniert" (Budin 2002: 84). Laut Budin (2002: 74) sind die für das Fachübersetzen wesentlichen Wissensarten das Sprach- und Kommunikationswissen, das Terminologiewissen, das translatorische Methodenwissen, das fachgebietsspezifische Sachwissen, das Informationsverwaltungs- und Managementwissen, das medientechnische Wissen, sowie das soziale und interkulturelle Handlungswissen. Hauptziel dieser Arbeit ist es, wie schon erwähnt, die Terminologie der Wirtschaftssprache mit einer konkreten Ausrichtung zum Fach Marketing zu erforschen, um die Fachübersetzung von wissenschaftlichen Texten im Bereich Marketing mit praktischen Erkenntnissen zu unterstützen. Das Terminologiewissen der Fachübersetzer soll somit für den Bereich Marketing intensiviert werden. Der Schwerpunkt liegt dabei auf den morphologischen und semantischen Aspekten der Terminologie des Marketings. Neben Komposita und Abkürzungen verschiedener Art werden auch Entlehnungen untersucht. Bei der Analyse der semantischen Aspekte werden Polysemie, Antonymie und Synonymie der Terminologie des Marketings sowie der Prozess der Terminologisierung betrachtet.

\section{Fachsprache}

Beim Betrachten der Fachsprache lassen sich viele Ansätze hervorheben, die jedoch keine voneinander unabhängigen, sondern stets miteinander im Zusammenhang stehende Forschungsgebiete darstellen. Bei einem Ansatz gibt man der Fachsprache eine auf das Fach und das Verwendungsziel verweisende Definition; der zweite stellt die Fachsprache der Gemein- oder Standardsprache gegenüber; beim dritten Ansatz wird die Fachsprache aus der Perspektive von Eigenschaften der verwendeten Sprachmittel betrachtet. Die Mehrheit der von einander ergänzenden Definitionen veranschaulicht den hohen Grad der Erarbeitung des Fachsprachenbereiches (vgl. Bausch 1976; Beier 1980; von Hahn 1983; Fluck 1985; Hoffmann 1987; Birkenmaier 1991; Cabré 1999 u. a.).

Eine breite und vielseitige Beschreibung der Fachsprache wird im Handbuch zur Sprach- und Kommunikationswissenschaft (HSK Halbbd. Fachsprachen 1998) vorgestellt. Hier wird auf verschiedene Aspekte und Felder der Fachsprachenforschung detailliert eingegangen, wie z.B. auf allgemeine Aspekte der Fachkommunikation, auf Auffassungen vom Status der Fachsprachen, Methoden in der Fachsprachenforschung, ihre Geschichte sowie auf ihre Ergebnisse. Ferner werden Verwendungs- und Systemeigenschaften von Fachsprachen (textuelle, lexikalisch-semantische, graphematische und phonologische Eigenschaften) beschrieben. Weitere Kapitel beschäftigen sich 
mit textlinguistischen Ansätzen. Eine nicht geringe Rolle spielen spezielle Aspekte der Fachkommunikation, wie z.B. die Verwendung von Fachsprachen in der modernen Arbeitswelt, Fachsprachen im inner- und zwischensprachlichen Kontakt, etc.

Die Fachsprache, einerseits als Widerspiegelung der im Entwicklungsprozess bereits erreichten Ergebnisse und andererseits als Mittel, die erworbenen Erkenntnisse weiterzugeben, dient dem Zweck, eine möglichst effektive, präzise und ökonomische Verständigung unter allen möglichen Kommunikationspartnern über ein bestimmtes Fachgebiet zu gewährleisten (Schmitt 1985: 18). Durch alle Entwicklungsphasen der Fachsprache zieht sich im Hintergrund der Kommunikationsprozess wie ein roter Faden (vgl. Bausch, Schewe \& Spiegel 1976: 12; Möhn \& Pelka 1984: 26; Hoffmann 1982: 2; Hoffmann 1993: 614). Das von Roelcke vorgestellte fachsprachliche Kommunikationsmodell veranschaulicht, welche Grundelemente und deren Verknüpfungen im Kommunikationsprozess zu berücksichtigen sind. In seinem Modell fachsprachlicher Kommunikation unter Berücksichtigung der Interessenschwerpunkte verschiedenartiger Fachsprachenkonzeptionen hebt der Verfasser das systemlinguistische Inventarmodell, das pragmalinguistische Kontextmodell und das kognitionslinguistische Funktionsmodell besonders hervor (vgl. Roelcke 2010: 13f.).

\section{Wirtschaftssprache und Fachsprache des Marketings}

Der Prozess der Globalisierung spiegelt sich in allen menschlichen Tätigkeiten wider und der Wirtschaftsbereich ist davon am meisten betroffen. Dies hat dazu geführt, dass wirtschaftliche Begriffe nicht nur in der professionellen Tätigkeit, sondern auch im Alltagsleben sehr verbreitet sind. All das veranlasst uns dazu, über die besondere Rolle der Wirtschaftssprache zu sprechen.

Schon in der ersten Hälfte des 20. Jahrhunderts wurde betont, dass das aktive Schaffen von Termini ein Merkmal des wirtschaftlichen Wachstums sei. Besonders intensiv wurden die terminologischen Forschungen in den sich schnell entwickelnden europäischen Ländern durchgeführt. Dieses Phänomen wird in der deutschen Sprache besonders deutlich, da Deutschland zu den Ländern gehört, deren Entwicklungstempo von Industrie und neuen Technologien am schnellsten ist. Bestimmte wirtschaftliche und politische Schwierigkeiten während der Entwicklung Spaniens im zwanzigsten Jahrhundert haben dazu geführt, dass viele wissenschaftliche, technische und wirtschaftliche Termini durch den Austausch zwischen Dolmetschern und Übersetzern entstanden sind. Die für die internationalisierten Branchen gebrauchte Terminologie wurde aus anderen Sprachen, insbesondere aus dem Englischen entlehnt. 
Hundt nennt zwei getrennte Phasen der wirtschaftssprachlichen Forschungen: die sogenannte Wirtschaftslinguistik der ersten Hälfte des 20. Jahrhunderts, und die Forschungen der Wirtschaftssprachen nach dem Zweiten Weltkrieg (vgl. Hundt 1998: 1296). In den letzten Jahren wurden verschiedene Aspekte der Wirtschafts- bzw. Unternehmenskommunikation unter besonderer Betrachtung der interkulturellen Aspekte erforscht (vgl. Baumann 2000; Bolten 1998; Bolten 2006; Brünner 2000; Bungarten 1996; Müller 1991). Es wurden aber sehr wenige Versuche unternommen, eine Typologie der Wirtschaftssprache zu erarbeiten (vgl. Bolten 1991; Hundt 1995 u.a.). Da der Begriff Wirtschaft an sich nicht homogen ist, lässt sich die Wirtschaftssprache als Kommunikationsmittel sehr schwer strukturieren. Nach Bolten (1991) lässt sich die Wirtschaftssprache vertikal in die Schichten Theoriesprache, Berufssprache und fachbezogene Umgangssprache einteilen (vgl. Bolten 1991: 75).

Diese Typologie wird von Hundt kritisiert (Hundt 1995: 51f.). Er unternimmt den Versuch, eine Mikrotypologie der Wirtschaftssprache vorzuschlagen. Dazu werden die institutionelle und die wissenschaftlich/theoretische Wirtschaftskommunikation analysiert. Für die erste übernimmt er als Kriterium das Drei-Sektoren-Modell. Es wird davon ausgegangen, dass drei Sektoren in der Gesamtwirtschaft auszumachen sind. Jeder dieser drei Sektoren kann je nach Entwicklungsgrad der Volkswirtschaft einen größeren oder einen kleineren Anteil ausmachen (vgl. Hundt 1995: 65). Für den zweiten Typ der Wirtschaftskommunikation scheinen ihm die Fachsprachen der Volksund Betriebswirtschaftslehre am wichtigsten zu sein. Sie bilden den Kern der Fachsprachen der Wirtschaftswissenschaften. Werden weitere Nachbarwissenschaften einbezogen, können weitere Fachsprachen der Wirtschaftswissenschaften differenziert werden, z.B. die Fachsprache der Wirtschaftsmathematik oder die Fachsprache der Wirtschaftsinformatik (ebd. 67-69).

Das Marketing ist eine Subdisziplin der Betriebswirtschaftslehre. Die folgende Definition verdeutlicht das Konzept und die zentrale Philosophie des Marketings: „In der klassischen Interpretation bedeutet Marketing die Planung, Koordination und Kontrolle aller auf die aktuellen und potenziellen Märkte ausgerichteten Unternehmensaktivitäten. Durch eine dauerhafte Befriedigung der Kundenbedürfnisse sollen die Unternehmensziele verwirklicht werden" (Meffert 2004, nach Meffert 2015: 12). Weitere zentrale Ideen des Marketings, sowie seine Ziele und Funktionen, auf die wir uns in diesem Beitrag stützen, wurden in den letzten zwei Jahrzehnten umrissen (Kuß 2013; Meffert 2015; Becker 2013; Bruhn 2014; Homburg 2012; Wöhe 2012 u.a.). 
Marketing ist ein komplexes System der Herstellungsorganisation und des Produktionsabsatzes, das auf die Befriedigung der Bedürfnisse von Kunden und auf Gewinn orientiert ist. Das wird durch Marktforschung und Marktprognose, Forschung der intrinsischen und extrinsischen Faktoren, Erarbeitung der Strategie auf dem Markt sowie durch Marketingprogramme erreicht. In die Marketingtätigkeit sind nicht nur Prozesse, die mit der Herstellung oder dem Absatz zu tun haben, sondern auch psychologische Prozesse, die auf die Entscheidung von Konsumenten wirken, eingeschlossen.

Die Fachsprache des Marketings sowie die des Managements sind kaum untersucht worden. Die Zahl der Werke, die sich mit diesen Themen beschäftigen, ist sehr überschaubar. Die Fachsprache des Marketings weist nicht nur gewöhnliche Komponenten einer Wirtschaftssprache auf, sondern auch Merkmale der wirtschaftlichen Psychologie (vgl. Hundt 1998: 1303), die auf das Marketing als Philosophie der Unternehmensführung zurückgeführt werden können (vgl. Bruhn 2014: 14-15).

\section{Korpora und die Extraktion der Wirtschaftsterminologie}

In dieser Arbeit ziehen wir ein vergleichbares Korpus für das Sprachenpaar Spanisch-Deutsch zur Analyse. Die analysierten Korpora bestehen aus Artikeln, die im Bereich Marketing veröffentlicht wurden. Das deutsche Korpus besteht aus allen Artikeln,die im Zeitraum zwischen 2000 und 2012 in der Fachzeitschrift „Der Markt - International Journal of Marketing“ erschienen sind. Das deutsche Korpus besteht aus 903.430 Wörtern. Im Falle des spanischen Korpus handelt es sich um alle im Zeitraum zwischen 2004 und 2014 in der Fachzeitschrift "Revista española de Investigación de Marketing“ erschienenen Artikel. Das spanische Korpus besteht aus 1.279.954 Wörtern.

Für die Extraktion von Termini haben wir die Software Terminus 2.0 benutzt, die von der Forschungsgruppe IULATERM (Universität Pompeu Fabra, Barcelona) erarbeitet wurde. Um höchst repräsentative Ergebnisse zu erreichen, wurden zwei Extraktoren (extractores) zunächst heruntergeladen und im Laufe der Extraktion angewandt. Die Extraktoren stellen eine Menge von Termini und terminologischen Wortverbindungen jeweils in jeder analysierten Sprache vor, die im wirtschaftlichen Bereich vorkommen. Sie wurden von uns vorbereitet und aus verschiedenen Quellen (einsprachige und zweisprachige Fachwörterbücher, Glossare, etc.) zusammengefasst. Dem Forschungsziel angemessen wurde der Extraktor durch weitere Termini und professionelle Redewendungen aus dem Bereich Marketing erweitert. Der Extraktor für das spanische Korpus besteht aus 9.360 Einheiten, der Extraktor für das deutsche Korpus besteht aus 9.945 Einheiten. Die repräsentative 
Menge soll bei ca. 2.500 terminologischen Einheiten liegen. Um den Analyseprozess zu gewährleisten, muss der Extraktor im Format TXT (UTF-8) angewandt werden. Die Wirtschaftstermini, die die Extraktoren enthalten, wurden von der Software nach morphologischen, lexikalischen und syntaktischen Kriterien analysiert. Dadurch ergibt sich ein Modell, das in der Software gespeichert und ferner für die Extraktion der Termini aus den wirtschaftswissenschaftlichen Texten verwendet wird. Ob der Prozess erfolgreich abgeschlossen wurde, ist auf Bild 1 ersichtlich.

Das Festlegen des Korpus stellt eine weitere Etappe der korpusbasierten Analyse dar. Nach dem Bearbeiten der Texte aus dem Korpus, sucht Terminus 2.0 Kandidaten für Termini und stellt sie in Form einer Tabelle vor. Neben der im Text vorhandenen Form wird auch das Lemma gezeigt. Die Kandidaten für Termini werden nach der Vorkommenshäufigkeit und nach dem kalkulierten Gewicht (ponderación) rangiert. Dieses Zwischenergebnis erlaubt, die Kandidaten zu evaluieren und somit eine Liste von Termini und terminologischen

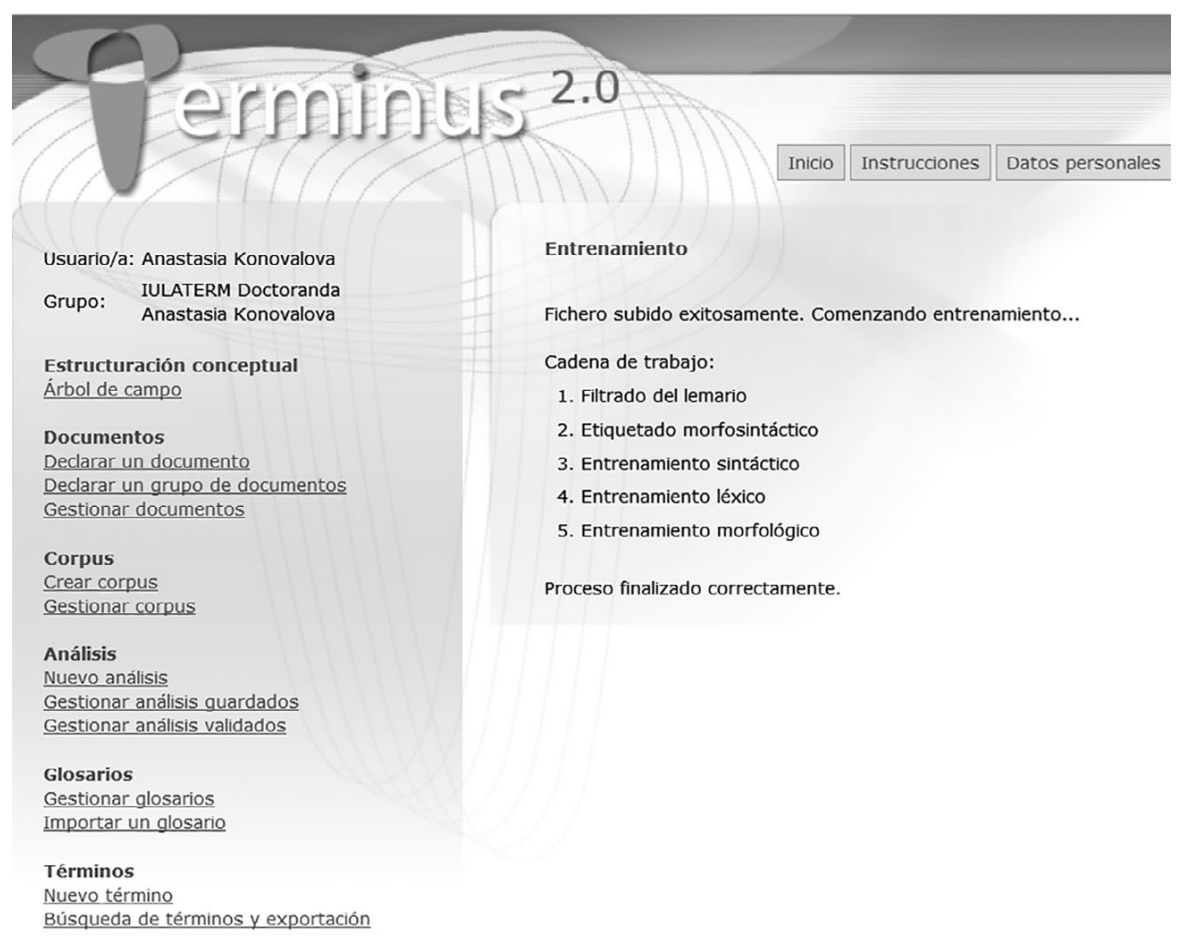

Bild 1. Das Herunterladen des Extraktors ist erfolgreich abgelaufen und alle Modelle von Termini wurden gespeichert 


\begin{tabular}{|c|c|c|c|c|c|}
\hline \multicolumn{3}{|c|}{$\begin{array}{l}\text { Volver } \\
\text { Validar candidatos seleccionados }\end{array}$} & \multirow[b]{2}{*}{ Lema } & \multirow[b]{2}{*}{ Frecuencia } & \multirow[b]{2}{*}{ Ponderación } \\
\hline Rank & & Forma & & & \\
\hline 1 & $\nabla$ & investigación de marketing & investigación de marketing & 1491 & 7905532.943700 \\
\hline 2 & $\nabla$ & relación & relación & 2410 & 684149.231615 \\
\hline 3 & $\nabla$ & transacción & transacción & 66 & 368904.318975 \\
\hline 4 & $\square$ & condición & condición & 124 & 260327.714702 \\
\hline 5 & $\nabla$ & intención de compra & intención de compra & 278 & 246717.793625 \\
\hline 6 & $\nabla$ & percepción & percepción & 454 & 234250.117336 \\
\hline 7 & $\nabla$ & motivación & motivación & 167 & 223055.600466 \\
\hline 8 & $\square$ & acción & acción & 102 & 183630.824415 \\
\hline 9 & $\nabla$ & promoción monetaria & promoción monetario & 63 & 168563.858546 \\
\hline 10 & $\square$ & orientación & orientación & 1224 & 153209.039888 \\
\hline 11 & $\nabla$ & inversión & inversión & 114 & 113692.138094 \\
\hline 12 & $\square$ & restricción & restriccion & 47 & 107766.553115 \\
\hline 13 & $\nabla$ & flujo & flujo & 213 & 106980.061160 \\
\hline 14 & $\square$ & atribución & atribución & 57 & 105936.445862 \\
\hline 15 & $\nabla$ & investigación en marketing & investigación en marketing & 19 & 100812.508647 \\
\hline 16 & $\nabla$ & distribución comercial & distribución comercial & 19 & 93633.910225 \\
\hline 17 & $\square$ & efecto & efecto & 1914 & 92272.103592 \\
\hline 18 & $\nabla$ & satisfacción & satisfacción & 1318 & 90032.322114 \\
\hline 19 & $\square$ & función & función & 540 & 88577.159547 \\
\hline 20 & $\square$ & repetición publicitaria & repetición publicitario & 24 & 88297.619335 \\
\hline 21 & $\nabla$ & relación de producto & relación de producto & 39 & 86881.002079 \\
\hline 22 & $\square$ & marketing science & marketing science & 480 & 84408.817486 \\
\hline 23 & $\square$ & medición & medición & 348 & 81222.618122 \\
\hline 24 & $\square$ & interacción & interacción & 309 & 79519.283330 \\
\hline 25 & $\square$ & cuota & cuota & 300 & 76789.442958 \\
\hline
\end{tabular}

Bild 2. Evaluierung der Termini, die von der Terminus 2.0 herausgefunden wurden

Einheiten zu erstellen, die für unsere Forschung relevant ist. Ein Teil der Evaluierung anhand der Beispiele aus dem spanischen Korpus ist auf dem Bild 2 dargestellt.

Bei der Evaluierung besteht die Möglichkeit, den Terminus im Kontext zu beobachten. In schwierigen Fällen wurde in zusätzlichen Quellen recherchiert, ob die Einheit in einer terminologischen Bedeutung oder in einer allgemeinsprachlichen Bedeutung vorkommt. Weist die gefundene Einheit nur die allgemeinsprachliche Bedeutung auf, wird sie aus der Liste der Kandidaten nicht hervorgehoben und ferner nicht betrachtet. Die daraus resultierende Analyse der allgemeinen, morphologischen und semantischen Aspekte der Wirtschaftsterminologie des Marketings stellen wir in den folgenden Punkten dieses Beitrages vor. 


\section{Terminologie des Marketings}

\subsection{Allgemeine Aspekte}

Zunächst ist die Wirtschaftsterminologie zu beobachten, die in allen Wirtschaftssprachen unabhängig von der Branche ihre Verwendung findet. Das ist die sogenannte wirtschaftliche Basisterminologie. Über diese wirtschaftlichen Begriffe werden die grundlegenden wirtschaftlichen Erscheinungen und Prozesse benannt. Für die deutsche und spanische Fachsprache des Marketings sind diese Termini im gleichen Maße charakteristisch, wie zum Beispiel:

De.: Großanbieter, Außenwirtschaft, Finanzierung, Hyperinflation, Wettbewerb, Produktionsfaktoren, Investitionsgüter, Unternehmensführung, Kostenhöhe, Umsatzhöhe, Endgewinn, Absatzmarkt, etc.

Es.: posición competitiva, comercialización, rendimiento, consumo, creación de valor, organización industrial, valor de transacción, competencia, reducción de costes, planificación, volumen de ventas, precio de compra, fabricación, costes de adquisición, costes de producción, factores de inflación, etc.

Die Auswahl der verwendeten terminologischen Einheiten ist nicht nur durch die Besonderheiten der Wirtschaftssprache geprägt, sondern auch durch die Textsorte. Der wirtschaftswissenschaftliche Artikel besteht aus verschiedenen Textsortenvarianten: Experimentalstudie, Fallbericht, Artikel zum Forschungsstand und andere. Diese Textsortenvarianten des wirtschaftswissenschaftlichen Beitrages bestimmen die Verwendung von Termini, die nicht nur aus der Wirtschaftsterminologie, sondern auch aus der Fachsprache der Statistik und der Mathematik stammen.

De.: Varianzanalyse, Varianzhomogenität, Varianzheterogenität, Stichprobenumfang, Prädiktorgröße, Korrelationskoeffizient, Indikatorreliabilität, etc.

Es.: modelización, ecuación, correlación, regresión, varianza, escala de medición, coeficientes de correlación, coeficientes de regresión, correlación parcial, función lineal, etc.

Neben den Wirtschaftstermini und den Termini der Fachsprache der Mathematik ist auf spezifische Termini und Wortverbindungen hinzuweisen, die für die Fachsprache des Marketings typisch sind und ihren Kern bilden. Marketing ist eine wichtige Komponente der Wirtschaftstätigkeit eines Unternehmens, um erfolgreich zu agieren. Der Kunde trifft eine Entscheidung zugunsten der Waren und Dienstleistungen, die er erwerben möchte, um seine Konsumbedürfnisse zu befriedigen. Der Herstellungssektor reagiert auf diese Bedürfnisse mittels der Herstellung der Waren und Dienstleistungen zu 
angemessenen Preisen. Um diese Prozesse und Begriffe in der Fachsprache des Marketings zu benennen, werden im Bereich Marketing zahlreiche lexikalische Einheiten verwendet, die viel mit den psychologischen Reaktionen und mit dem Verhältnis von realen und potenziellen Kunden verbunden sind. Die psychologisch und emotionell gefärbten Einheiten sind für die Fachsprache des Marketings unabdingbar. Je aufmerksamer man mit Konsumenten umgeht, desto größer ist die Chance, mehr Produkte zu verkaufen und somit mehr Gewinn zu erbringen.

Spezifische Kernbegriffe des Marketingbereichs lassen sich thematisch systematisieren. Sie befinden sich im Zusammenhang und wirken wechselweise aufeinander. Das Marketing an sich ist ohne Marktwirtschaft nicht vorstellbar.

Damit gelangen wir zum wichtigsten Begriff der Marketingphilosophie, nämlich dem Markt. Dabei handelt es sich nicht um den Markt als isolierten Begriff, sondern um alle vorstellbaren Aktivitäten, die Unternehmen auf dem Markt vornehmen. Für die präzisere Bezeichnung von solchen Aktivitäten und Maßnahmen, sowie um den Markt zu charakterisieren, lassen sich folgende Komposita in den analysierten Marketingtexten nachweisen":

$\begin{array}{ll}\text { Marktattraktivität } & \begin{array}{l}\text { atractividad/atractivo de mercado } \\ \text { orientado hacia el mercado } \\ \text { marktorientiert }\end{array} \\ \text { Marktsättigung } & \text { sabjeto del mercado mercado } \\ \text { Marktgegenstand } & \text { objer }\end{array}$

Der Bereich Marketing ist vor allem als kundenorientiert zu bezeichnen, deshalb beschäftigen sich Wirtschaftswissenschaftler aus diesem Bereich mit Begriffen wie z.B. Kunden, Konsumenten und Käufer. Um die Verhältnisse zu diesen Verwaltungsobjekten zu bezeichnen, werden zahlreiche Komposita mit den entsprechenden Basiselementen verwendet:

Käuferschwund
Käuferreaktion
Käuferverhalten pérdida de compradores

reacción de compradores

comportamiento de compradores

1. Hier und ferner werden deutsche und spanische Beispiele tabellarisch vorgestellt. Dabei wurden die deutschen Beispiele dem deutschen Korpus und die spanischen Beispiele sowohl dem spanischen Korpus und als auch Internet-Quellen entnommen, d.h. die zweite Spalte der Tabelle schlägt teilweise die Übersetzungen der deutschen Terminologie vor. 
Konsumentenloyalität

Konsumentenverhalten

Konsumentenforschung

Kundenadaptivität

Kundenzufriedenheit

Kundenkomplexität lealtad de los consumidores comportamiento del consumidor investigación de consumidores adaptabilidad del cliente satisfacción del cliente complejidad de los clientes

Auf dem Markt bietet man den Kunden verschiedene Produkte und Dienstleistungen an. Folgende Komposita treten am häufigsten auf:

Produktattraktivität
Produktkategorien
Produktidentität
Produktqualität
Produktproben
Produktwerbung
Gütermarkt
Güterbeschaffung
Gütertransport
Güterbereich
Gütereigenschaften
Gütertransfer
Güterfluss

atractividad/atractivo del producto

categorías del producto

identidad del producto

calidad del producto

prueba del producto

publicidad del producto

mercado de mercancías

adquisición de mercancías

transporte de mercancías

campo/sector de mercancías

características de las mercancías

transferencia de mercancías

flujo de mercancías

Ein weiterer wichtiger Begriff im Bereich Marketing ist Marke. Um diesen Begriff möglichst präzise zu bezeichnen, werden folgende Komposita verwendet:

Markenname
Markenartikel
Markenbekanntheit
Markentransfer
Markenbewertung
Markenpolitik
Markenbeziehungsqualität
Markenwert
Markenattraktivität
Markenoriginalität
Markenführung

nombre de la marca

artículo de marca

fama de la marca

transferencia de la marca

evaluación de la marca

política de marca

calidad, relacionada con la marca

valor de la marca

atractividad de la marca

originalidad de la marca

gerencia de marca 
Wie man aus den angeführten Beispielen feststellen kann, betrachtet man die Marke als ein Instrument, um mehr Kunden anzuziehen. Weitere Instrumente, die ihre Verwendung bei der Kundenbeziehung finden, sind Qualität und Preis, die in den meisten Fällen in Kombination auftreten:

Preisdarstellung
Preisdifferenzierung
Preispartitionierung
Preisattraktivität
Produktqualität
Dienstleistungsqualität
Servicequalität
Relationsqualität
Mindestqualität

indicación del precio

diferenciación de precios

precio desglosado

atractividad del precio

calidad del producto

calidad de servicios

calidad de servicios

calidad de relaciones

calidad mínima

Die verwendeten Marketinginstrumente sollen auf die Reaktionen von Kunden wirken, unter anderem auf ihre Präferenzen. Dabei ist es ein gutes Zeichen, wenn der Kunde der Marke „treu“ bleibt:

Präferenzschaffung
Präferenzbedeutung
Präferenzangebot
Präferenzmessmethoden
Präferenzvorteile
Präferenzwerte
Markenloyalität
Beraterloyalität
Kundenloyalität

formación de preferencias significado/importancia de preferencias oferta beneficiosa métodos de la medida de preferencias ventajas de preferencias valores de preferencias

lealtad a la marca lealtad al consultor lealtad del cliente

5.2. Morphologische Aspekte: Komposita in der deutschen Fachsprache des Marketings und ihre Äquivalente im Spanischen

Bei der vergleichenden Untersuchung der Wortbildungssysteme des Deutschen und Spanischen in den Texten des Korpus wurde festgestellt, dass die vorkommenden Strukturen sich sehr stark voneinander unterscheiden. Während für die deutsche Fachsprache des Marketings die häufige Verwendung von Komposita charakteristisch ist, bevorzugt die spanische Sprache an erster Stelle präpositionale Wortgruppen. Laut Birkenmaier (1991: 33) drücken Komposita, auch Mehrworttermini genannt, „einen einzigen Begriff gegliedert 
aus. Durch eine solche sprachliche Gegliedertheit wird die innere Form des Begriffs wiedergegeben, so dass auf dem Wege einer begrifflichen Etymologie die Zusammenhänge und Beziehungen mit anderen Begriffen dargelegt werden". Präpositionale Wortgruppen dagegen bringen die Beziehung zwischen den einzelnen Gliedern der Gruppe mithilfe einer Präposition zum Ausdruck.

Aus den in verschiedenen Werken zur Sprachwissenschaft aufgelisteten Kompositatypen (Determinativkomposita, Possessivkomposita, Kopulativkomposita) (vgl. Motsch 2004; Eichinger 2000; Donalies 2007 u.a.) kommt in den analysierten Korpora mit einer hohen Frequenz ein Typ vor, und zwar das Determinativkompositum. Bei Determinativkomposita hat das Erstelement eine modifizierende Funktion, d.h., die semantische Repräsentation des Zweitelementes wird durch die semantische Repräsentation des Erstelementes ergänzt (vgl. Motsch 2004: 376). Dieser Kompositatyp kommt in der Fachsprache des Marketings am häufigsten vor, weil Wirtschaftsbegriffe immer wieder präzisiert werden müssen.

Die herausgefundenen Determinativkomposita sind vor allem Substantive und lassen sich mithilfe Eichingers Subklassifizierung untergliedern (vgl. Eichinger 2000: 118-123). Das erste Modell der substantivischen Determinativkomposita, auch genitivische Komposita genannt, ist das N+N-Kompositum und hat als erste Konstituente die Form eines genitivischen Attributs. Die Umformung eines Kompositums in eine genitivische Wortgruppe ist die häufigste Form, in die sich die unmittelbaren Konstituenten auflösen lassen (z.B. Marketingaktivität $\rightarrow$ Aktivität des Marketings). Dies hat zufolge, dass die Entsprechungen solcher Komposita im Spanischen mit der präpositionalen Wortgruppe $\mathrm{N}+d e$ (art.) $+\mathrm{N}$ wiedergefunden werden, denn in den meisten Fällen handelt es sich um ein Genitivus possessivus nach der Gliederung von Helbig/Buscha (2001: 497f) und der wird auf Spanisch mit der Präposition de gebildet, z.B.:

$$
\begin{array}{ll}
\text { Marketingaktivität } \rightarrow \text { Aktivität des Marketings } & \text { actividades de marketing } \\
\text { Kundenadaptivität } \rightarrow \text { Adaptativität der Kunden } & \text { adaptabilidad de clientes } \\
\text { Gütertransfer } \rightarrow \text { Transfer der Güter } & \text { transferencia de } \\
& \text { mercancías } \\
\text { Markentransfer } \rightarrow \text { Transfer der Marken } & \text { transferencia de marcas }
\end{array}
$$

Ein weiteres Modell ist die Verbindung von Adjektiven mit Nomina (Adj. + $\mathrm{N}$ ). Je nach der Zahl von Elementen, die das deutsche Kompositum bilden, können diese Einheiten im Spanischen als eine adjektivische Wortgruppe mit der Struktur Adj. + N erscheinen (z.B. gran consorcio), wobei die Reihenfolge der Konstituenten erhalten bleibt und das Adjektiv nicht eine restriktive 
(wie bei den nachgestellten Adjektiven), sondern vielmehr eine erläuternde Funktion hat. Sie können aber auch als einfache präpositionale Wortgruppe $(\mathrm{N}+d e+\mathrm{N}, \mathrm{usw}$.) oder als erweiterte präpositionale Wortgruppe mit verschiedenen Strukturen $(\mathrm{N}+\mathrm{con}+\mathrm{A}+\mathrm{N}, \mathrm{N}+\mathrm{de}+\mathrm{N}+\mathrm{A}$, usw.) erscheinen.

Beispiele von Komposita mit den Komponenten Groß- oder Außen-:

Großverdiener

Großkonzern

Großkunden

Großunternehmen

Außendienstpersonal

Außenwirtschaftsorganisation

Außenhandelsdaten persona con altos ingresos

multinacional

cliente preferente

empresa grande

personal de ventas

organización de economía exterior

datos de comercio exterior

Bei den deutschen wirtschaftswissenschaftlichen Texten sind neben Zweikomponentenkomposita auch Dreikomponentenkomposita vorhanden, die sowohl aus terminologischen, als auch aus allgemeinsprachlichen Einheiten bestehen und nach verschiedenen Modellen gebildet werden, z. B.:

Erfolgsfaktorenqualität

Eigenkapitalrentabilität rentabilidad de los factores de éxito

rentabilidad del capital propio

Die gefundenen spanischen Entsprechungen sind Kombinationen aus präpositionalen und adjektivischen Wortgruppen mit verschiedenen Strukturen, die die Beziehungen der Konstituenten zueinander darstellen $(\mathrm{N}+$ de (art.) + $\mathrm{N}+d e+\mathrm{N}$, für das erste Beispiel und $\mathrm{N}+$ de $(\operatorname{art})+.\mathrm{N}+$ Adj. für das zweite Beispiel).

Solche Einheiten stellen normalerweise mehr Schwierigkeiten im Übersetzungsprozess dar als die Zweikomponentenkomposita, weil die Chancen, eine angemessene Lösung in Wörterbüchern zu finden, geringer sind und die Zusammenhänge zwischen den Konstituenten schwieriger festzustellen sind.

Einerseits ist es für die deutsche Fachsprache des Marketings zweifellos von Vorteil, dass sich eine große Informationsmenge in einer semantisch geräumigen Struktur konzentriert. Anderseits sind gewisse Schwierigkeiten beim Verstehen und beim Übersetzen dieser terminologischen Einheiten unvermeidbar. 


\subsection{Entlehnungen}

Das Marketing ist eine der wichtigsten Philosophien der Unternehmensführung, kennzeichnet die Marktwirtschaft und ist für alle Branchen und Unternehmenstypen, die nach den marktwirtschaftlichen Prinzipien agieren, gültig. Die auch heute berühmten und oft verwendeten Marketingkonzepte entstanden in der zweiten Hälfte des 19. Jahrhunderts und zu Beginn des 20. Jahrhunderts in den englischsprachigen Ländern als Reaktion auf die Entwicklung der industriellen Produktionsweise. Sie waren in verschiedenen Entwicklungsphasen des Marketing unterschiedlich ausgeprägt (vgl. Kuß 2013: 5; Meffert 2015: 6-10). Für die deutsche und die spanische Sprache gilt, dass viele Begriffe und damit verbundene Termini vorwiegend aus dem Englischen entlehnt wurden. Die oben erwähnten Faktoren haben zur Internationalisierung der englischen Begriffe in der Terminologie des Marketings der deutschen und der spanischen Sprache geführt. Um zu veranschaulichen, wie häufig Entlehnungen aus dem Englischen in den wirtschaftswissenschaftlichen Marketingtexten verwendet werden, zitieren wir einen kurzen Abschnitt aus dem deutschen Beitrag „Online-Marketing-Controlling - Neue Wege in der Erfolgsmessung von Marketingmaßnahmen".

Die besondere Problematik für die E-Tailer besteht in der Notwendigkeit, nicht nur starke Marken zu verkaufen, sondern sich selbst in der neuen Unübersichtlichkeit des WorldWideWeb als Retail Brand zu positionieren. In der Anfangsphase des B2C mit zahlreichen verschiedenen Geschäftskonzepten war es die wichtigste Aufgabe für die First Mover, die Besucherfrequenz auf ihren Seiten zu erhöhen und damit die Awareness für die Marke aufzubauen. So wurde die Anzahl der Hits, der Benutzerzahlen, schnell zur Maßzahl des Erfolgs und trieb die Marktkapitalisierung nach oben (Klacklauer 2001: 151).

Der angeführte Abschnitt weist zahlreiche Entlehnungen auf, sowohl zur allgemein gebrauchten Lexik, (WorldWideWeb, Awareness, Hits) als auch zur Wirtschaftsterminologie des Marketings (E-Tailer, Retail Brand, B2C). Bereits im Titel des Beitrags steht eine Entlehnung Online-Marketing-Controlling. Aus dem Englischen wurden die Bezeichnungen für die Marketingfunktionen entlehnt, so werden in der deutschen und in der spanischen Sprache die Termini Marketing-Management, oder eine weitere deutsche Variante Marketingmanagement, verwendet.

In der Klassifizierung, die von J. Gómez Capuz in der Studie Préstamos del español: lengua y sociedad vorgenommen wurde, werden verschiedene Entlehnungstypen (graphische, morphologische, semantische und lexikalische) analysiert (Gómez Capuz 2004). Da es beim Entlehnungsprozess von 
Wirtschaftstermini fast immer um die Entstehung neuer terminologischer Einheiten geht (Form plus Bedeutung), lassen sich die erforschten fremdsprachlichen Einheiten als lexikalische Entlehnungen identifizieren, die in Importe, Hybride und Lehnübersetzungen eingeteilt werden. Beim Import handelt es sich um eine vollständige Entlehnung, bei der sich die terminologischen Einheiten nicht an die phonetischen, graphischen und grammatischen Besonderheiten der rezipierenden Sprache anpassen. Diese Erscheinung ist, abgesehen von geringfügigen Abweichungen, in der spanischen und der deutschen Sprache gleichermaßen typisch, z.B.:

De.: Marketing, Branding, Faktoring, Electronic Commerce/E-Commerce, Global-Marketing, International Marketing, Nonprofitmarketing;

Es.: marketing, branding, factoring, stocks, joint ventures, target pricing, Supply Chain Management.

Für die Benennung der klassischen und modernen Marketingberufe werden vor allem die Entlehnungen dieser Art verwendet: Marketing Manager, Product Manager, Sales Manager, Key Account Manager, Communications Manager, Market Research Manager, etc. Besonders präsent ist diese Art der Entlehnung bei den englischen Abkürzungen, die in den deutschen und spanischen wirtschaftswissenschaftlichen Texten verbreitet sind, z.B.:

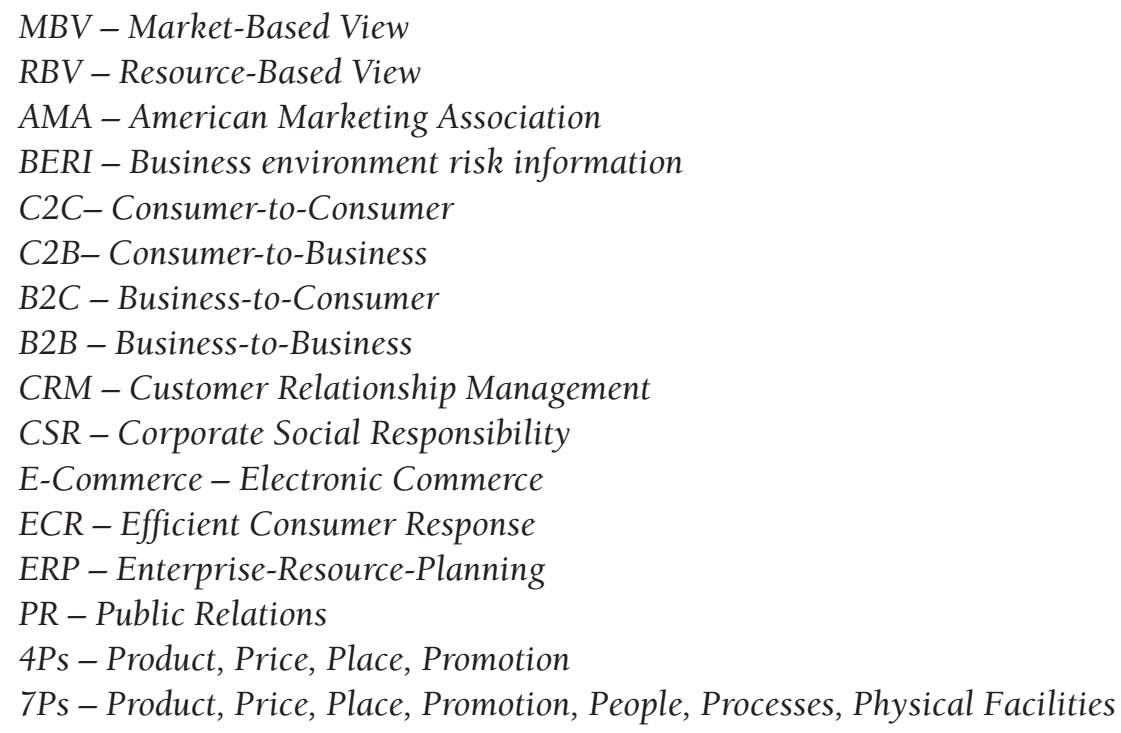

Bei den „Hybriden“ geht es um die aus mehreren Morphemen bestehenden Einheiten, wovon der eine Teil komplett entlehnt, der zweite durch eine 
Urform oder eine Lehnübersetzung ersetzt wird. In diesem Fall können sowohl die derivativen „Hybriden“ - z. B. der Stamm ist entlehnt, das Präfix oder das Suffix ersetzt - als auch die Komposita beobachtet werden. So sind im analysierten Korpus die Komposita mit der Entlehnung Marketing auffällig. Diese Einheit kann sowohl mit weiteren Entlehnungen als auch mit deutschen Wörtern verschiedene Komposita bilden. Im Spanischen wird die englische Einheit zusammen mit spanischen Wörtern in eine Wortverbindung zusammengestellt ${ }^{2}$.

$\begin{array}{lll}\text { Englisch } & \text { Deutsch } & \text { Spanisch } \\ \text { Marketing Research } & \text { Marketingforschung } & \text { investigación de marketing } \\ \text { Marketing Instruments } & \text { Marketinginstrumentarium } & \text { instrumentos de marketing } \\ \text { Direct Marketing } & \text { Direktmarketing } & \text { mercadotecnia directa } \\ \text { Social Marketing } & \text { Sozialmarketing } & \text { mercadotecnia social } \\ \text { Relationship Marketing } & \text { Beziehungsmarketing } & \text { mercadotecnia relacional } \\ \text { Experience Marketing } & \text { Erlebnismarketing } & \text { mercadotecnia experencial }\end{array}$

Bei der Lehnübersetzung wird eine aus mehreren Morphemen bestehende lexikalische Einheit durch schon in der rezipierenden Sprache existierende Morpheme oder durch einfachere Morpheme so ersetzt, dass die Verbindung von diesen Elementen als eine völlig neue lexikalische Einheit erscheint, die früher noch nicht bekannt war. Im Englischen ist das Modell „Definierendes (Attribut) + Definiertes (Objekt)"vorhanden, was im Spanischen mit der Veränderung der Reihenfolge von Wörtern durch das Modell „Definiertes + Definierendes" ersetzt wird. Im Deutschen spiegelt sich das englische Modell wider (die Reihenfolge wird beibehalten).

$\begin{array}{lll}\text { Englisch } & \text { Deutsch } & \text { Spanisch } \\ \text { RED-Research and } & \text { FEE-Forschung und } & \text { IED - Investigación y } \\ \text { Development } & \text { Entwicklung } & \text { Desarrollo } \\ \text { Customer Lifetime Value } & \text { Kundenlebenszeitwert } & \text { Valor de Vida del Cliente } \\ \text { External Analysis } & \text { unternehmensexterne Analyse } & \text { Análisis externo } \\ \text { Internal Analysis } & \text { unternehmensinterne Analyse } & \text { Análisis interno } \\ \text { Know How } & \text { Know How } & \text { Saber cómo/Know How } \\ \text { ICT - Information } & \text { IKT - Informations- und } & \text { TICs - Tecnologías de } \\ \text { and Communication } & \text { Kommunikationstechnologien } & \text { la Información y las } \\ \text { Technologies } & & \text { Comunicaciones }\end{array}$

2. Um die oben beschriebenen Zusammenhänge zu veranschaulichen, haben wir in dieser und in der nächsten Tabelle neben den deutschen Beispielen und ihren Äquivalenten im Spanischen auch die englischen Ausgangstermini angeführt. 


$\begin{array}{lll}\text { RBV-Resource-Based } & \text { ROA-Ressourcenorientierter } & \text { VBR - Visión basada en } \\ \text { View } & \text { Ansatz } & \text { Recursos } \\ \text { SWOT Analysis - } & \text { SWOT-Analyse } & \text { Análisis DAFO o Análisis } \\ \text { Strengths, Weaknesses, } & & \text { FODA (Debilidades, } \\ \text { Opportunities and } & & \text { Amenazas, Fortalezas y } \\ \text { Threats } & \text { Oportunidades) }\end{array}$

\subsection{Semantische Aspekte}

In der deutschen und spanischen Fachsprache des Marketings kommen Einheiten vor, die sowohl in der allgemeinen Sprache als auch in der Fachsprache verwendet werden. Das sind polysemantische Einheiten, die je nach dem Kontext verschiedene Bedeutungen ausdrücken können. Auf diese „Terminologisierung" wurde von Fluck hingewiesen. Einige Bezeichnungen werden aus der allgemeinsprachlichen Lexik übernommen und mit einer fachbezogenen Bedeutung versehen. Es wird keine neue Lautform, sondern eine neue Begriffsbedeutung geschaffen (vgl. Fluck 1984: 43).

In der Fachsprache des Marketings werden lexikalische Einheiten verwendet, die allgemeinsprachlich sind, aber im wirtschaftlichen Kontext eine terminologische Bedeutung erwerben können. Auf den ersten Blick kann nicht eindeutig festgestellt werden, mit welcher Bedeutung sie verwendet werden. Solche Einheiten bereiteten bereits in der Anfangsphase der Analyse mit der Software Terminus 2.0. bei der Evaluierung von Termini viele Schwierigkeiten. Sie sind besonders für die Beschreibung von Marktständen oder Kundenreaktionen typisch.

Ein Beispiel für dieses Phänomen mag die Einheit Sättigung/saturación sein. Das Wörterbuch Duden schlägt zwei Bedeutungen vor, eine davon ist das Sättigen, Stillen des Hungers; das Sattsein und die zweite ist das Sättigen, Gesättigtsein. Bereits im Duden wurde darauf hingewiesen, dass die zweite Bedeutung des Wortes für die Fachsprache geeignet ist. Die zweite Bedeutung ermöglicht es dieser lexikalischen Einheit, sich mit weiteren Einheiten zu verbinden und einen spezifischen wirtschaftlichen Sinn auszudrücken. So entsteht der Marketingbegriff Marktsättigung. Das spanische Diccionario de la Real Academia Española bietet nur eine Definition des Wortes saturación an, und zwar 1. Acción y efecto de saturar. Schlagen wir das Verb saturar nach, finden wir weitere Bedeutungen, einige davon sind terminologisch und für die Bereiche Physik oder Chemie typisch. Dem wirtschaftlichen Kontext nach würde die zweite Bedeutung passen: 2. saciar (hartar y satisfacer de comida $o$ de bebida). Man kann von der Sättigung mit Waren oder Dienstleistungen sprechen. 
Neben der Polysemie ist auch die Partonymie in der Fachsprache des Marketings präsent. Die partonymischen Verhältnisse verbinden die lexikalischen Einheiten nach dem Prinzip „ein Teil - das Ganze" oder "Menge Element der Menge". In der Fachsprache des Marketings sind auch solche Verbindungen zwischen Termini charakteristisch und können als Zyklus oder als Kette vorgestellt werden:

De.: Versorgung $\sim$ Herstellung $\sim$ Verteilung; Planung $\sim$ Organisierung $\sim$ Realisierung Kontrolle;

Es.: aprovisionamiento $\sim$ producción $\sim$ distribución; planificación $\sim$ organización $\sim$ realización $\sim$ control.

Aus der Gesamtheit von Antonymen fallen zunächst die Gruppen der Termini mit gegensätzlichen Bedeutungen auf, bei denen nur die am Rande gelegenen Einheiten als völlig kontrastiv identifiziert werden können:

De.: Anbieter $\sim$ Hersteller Vermittler Verbraucher;

Es.: proveedor $\sim$ fabricante $\sim$ intermediario $\sim$ consumidor.

In den analysierten Texten kommen Einheiten sehr häufig vor, die Verhältnisse zwischen den auf dem Markt agierenden Hauptakteuren ausdrücken. In den spanischen Texten sind Beispiele enthalten, in denen das Wort relación vorausgeht. In den deutschen Texten setzt man das Wort Verhältnis oder das Wort Beziehung vor. Nach diesem Wort werden zwei Wörter mit kontrastiver Bedeutung verwendet, die mit einem Bindestrich verbunden sind:

De.: Verkäufer-Konsumenten, Hersteller-Händler, Verbraucher-Unternehmen, Unternehmen-Konsument.

Es.: vendedor-cliente, vendedor-consumidor, comprador-proveedor, compradorvendedor, cliente-empresa, empresa-cliente, empresa-consumidor, etc.

Solche Strukturen spiegeln die Einwirkung der außersprachlichen Faktoren auf die Besonderheiten der Wirtschaftssprache wider. So werden die Wirtschaftsrealien und eine logische Vorstellung ihrer Funktionsweise in einer sehr komprimierten Form ausgedrückt. In der analysierten Wirtschaftsterminologie sind auch Antonyme vorhanden, die Merkmale oder Aktivitäten in verschiedenen Richtungen benennen. Bei der Analyse des Korpus wurde auch festgestellt, dass die Antonymie durch kontrastive Präfixe sehr häufig vorkommt. Dazu können auch weitere deutsche Komposita oder spanische Wortverbindungen angeführt werden, bei denen eine Komponente kontrastiv ist: 
De.: Import Export, Input Output, Kapitalabfluss Kapitalzufluss, Ausfuhr Einfuhr, Einkäufer Verkäufer, Einzahlung Auszahlung, materielle immaterielle, monetär nicht monetär, Aktivgeschäft Passivgeschäft, Bruttoumsatz Nettoumsatz, Einzelhandel Großhandel.

Es.: aprovisionamiento $\sim$ distribución, desvalorización $\sim$ revalorización, exportación importación, crecimiento $\sim$ decrecimiento de la economía, valorización desvalorización, rentabilidad $\sim$ irrentabilidad, materiales $\sim$ inmateriales, inversión directa inversión indirecta, comercio al por menor $\sim$ comercio al por mayor.

Die Antonymie durch die Gegensetzung von einzelnen Komponenten der Komposita ist für die deutsche Sprache charakteristisch. In der spanischen Sprache ist die Antonymie durch die Präfixe oder durch einen Teil der Wortverbindung am häufigsten.

\section{Schlussfolgerung}

In der Menge von Fachübersetzungen sind die Übersetzungen im wirtschaftlichen Bereich besonders hervorzuheben. Der Grund dafür ist die Wichtigkeit der Fachkommunikation in internationalen Unternehmen. Übersetzer sollen den Kommunikationsprozess zwischen den Spezialisten im wirtschaftlichen Bereich gewährleisten, was sehr oft eine große Herausforderung darstellt. Die Qualität der Übersetzung ist unter anderem davon abhängig, in wieweit sich der Übersetzer mit der Wirtschaftssprache auseinandergesetzt hat. In diesem Beitrag wurde die Terminologie des Marketings, besonders ihre morphologischen und semantischen Aspekte analysiert, um Schwierigkeiten beim Übersetzen von Marketingtexten zu erleichtern.

In der Terminologie des Marketings sind drei Kategorien hervorzuheben, die in den wirtschaftswissenschaftlichen Texten des Marketingbereichs vorkommen: die wirtschaftliche Basisterminologie, spezifische Termini und Wortverbindungen aus dem Bereich Marketing, sowie die Terminologie der Statistik und Mathematik. Je nach der Textsortenvariante und dem betrachteten Thema des wissenschaftlichen Beitrages kann diese Palette erweitert werden.

Der wissenschaftliche Stil und ein hoher Grad an Spezialisierung sind für die analysierten Texte kennzeichnend und beim Übersetzen besonders zu beachten. Um beim Übersetzen von solchen wirtschaftswissenschaftlichen Texten sicherzugehen, ist dem Übersetzer zu empfehlen, sich neben dem linguistisch orientierten Terminologiewissen auch das fachgebietsspezifische Sachwissen anzueignen. Die spezifische Terminologie des Marketings lässt sich thematisch je nach Hauptakteuren und Instrumenten des Marketings 
systematisieren. Das Marketing an sich ist ohne solche Begriffe wie Markt, Kunden, Konsumenten, Käufer, Produkt, Marke, Preis, Präferenz u.a. nicht vorstellbar. Versteht der Übersetzer die Zusammenhänge und die Zusammenwirkung dieser Marketingkategorien, fällt es ihm leichter, das Verhältnis zwischen den Termini zu verstehen und klar im Spanischen oder in einer anderen Sprache wiederzugeben.

Beim Betrachten der morphologischen Aspekte wurden folgende Besonderheiten der Terminologie des Marketings festgestellt: Für die deutsche Fachsprache des Marketings ist die häufige Verwendung von Komposita charakteristisch, was sich sehr stark von den spanischen Strukturen unterscheidet. Zwei- und Dreikomponentenkomposita wurden in den analysierten deutschen Marketingtexten gefunden. Dabei kommt ein Kompositatyp am häufigsten vor, nämlich Determinativkomposita verschiedener Typen $(\mathrm{N}+\mathrm{N}, \mathrm{A}+\mathrm{N}, \mathrm{N}+\mathrm{V})$. Die Verwendung der Determinativkomposita ist durch verschiedene Gründe verursacht. Einerseits besteht die Notwendigkeit, die Wirtschaftsbegriffe immer wieder zu konkretisieren (die schon vorhandenen Wirtschaftsbegriffe werden präzisiert). Andererseits können Termini dadurch entstehen, dass diese schon in die bereits vorhandene allgemeinsprachliche Einheit hinzugefügt werden. Obwohl bekannt ist, dass deutsche Komposita ins Spanische in den meisten Fällen mit verschiedenen Präpositionalphrasen (z.B. $\mathrm{N}+$ Präposition $+\mathrm{N}$ ) wiederzugeben sind, fiel es uns nicht leicht, die entsprechenden spanischen Äquivalente in der Terminologie des Marketings zu finden. Um solche Schwierigkeiten und Zeitverluste zu überwinden, ist es $z u$ empfehlen, eine eigene alphabetisch oder thematisch geordnete Liste von erarbeiteten Lösungen zu führen. Sehr hilfreich und zeitsparend ist die Verwendung verschiedener Softwares zur Terminologieverwaltung.

Da der Bereich Marketing in den englischsprachigen Ländern entwickelt wurde, wurden viele Begriffe und damit verbundene Termini vorwiegend aus dem Englischen entlehnt. Die durchgeführte Analyse der englischsprachigen Entlehnungen in der Wirtschaftsterminologie des Deutschen und des Spanischen hat deutlich gezeigt, dass die folgenden weitverbreiteten Arten des Übergangs der entlehnten Wörter in die rezipierende Sprache typisch sind: Importe, Hybride und die lexikalische Lehnübersetzung. Für die deutsche Sprache und für die spanische Sprache sind in höherem Maße alle drei Arten der Entlehnungen mit oder ohne phonetischen und graphischen Abweichungen nachzuweisen. Beim Übersetzen ist aber zu beachten, dass die gewählte Einheit den orthographischen Normen der rezipierenden Sprache angepasst wird. Manchmal sind verschiedene Übersetzungs- und Schreibvarianten derselben Einheit zu finden. An solchen Stellen wäre ein Wörterbuch von 
Entlehnungen oder Neologismen mit den entsprechenden Erklärungen und Hinweisen zur Benutzung sehr hilfreich. Auch Internet-Quellen sind hierfür nützlich. Durch eine kurze Suche nach terminologischen Einheiten in elektronischen Suchmaschinen kann festgestellt werden, welche Vorkommenshäufigkeit jede Variante aufweist.

Aus der Analyse der semantischen Aspekte der Marketingterminologie können folgende Zusammenhänge zum Übersetzen festgestellt werden: Eine gewisse Schwierigkeit stellt die Wiedergabe von lexikalischen Einheiten dar, die zwar allgemeinsprachlich sind, aber in wirtschaftswissenschaftlichen Beiträgen eine terminologische Bedeutung bekommen können. Um mit solchen Einheiten sicherer umzugehen, können für den Übersetzer sowohl die Definitionen aus einem Wörterbuch, als auch der wirtschaftliche Kontext sehr hilfreich sein. Sehr oft werden polysemantische Einheiten durch weitere terminologischen Einheiten konkretisiert, so dass ein spezifischer wirtschaftlicher Sinn ausgedrückt wird.

Eine weitere Schwierigkeit beim Fachübersetzen von wirtschaftswissenschaftlichen Texten im Bereich Marketing wird vor allem beim Übersetzen der Terminologie des Marketings deutlich, wenn diese eine psychologische oder emotionelle Färbung in sich trägt. Nicht in allen Fällen können dieselben psychologischen Aspekte identisch wiedergegeben werden. Der Grund dafür ist die verschiedene Art und Weise der kulturellen Wahrnehmung dieser Aspekte in den verschiedenen Sprachen. Stets sind verschiedene spezifische Merkmale zu finden und beim Vergleich der Wirtschaftssprachen können sich die Unterschiede auf allen sprachlichen Ebenen manifestieren. Diese Unterschiede können durch Beratungen von Spezialisten, die sich jeden Tag mit solchen Begriffen beschäftigen und sie sicher beherrschen, aufgeklärt und gelöst werden.

\section{Literatur}

BAUMANN, Klaus-Dieter. (2000) „Unternehmenskommunikation und Unternehmensidentität aus kommunikativ-kognitiver Sicht." In: Morgenroth, Klaus (Hrsg.) 2000. Hermetik und Manipulation in den Fachsprachen. Reihe „Forum für Fachsprachenforschung“. Band 55. Tübingen: Gunter Narr Verlag, S. 107-126.

BAUSCH, Karl-Heinz (Hrsg.) (1976) Fachsprachen. Terminologie, Struktur, Normung. Berlin \& Köln: Beuth Verlag GmbH.

BAUSCH, Karl-Heinz; Wolfgang Schewe \& Heinz-Rudi Spiegel. (1976) „Eindeutige Verständigung - ein Element technisch-wissenschaftlicher Entwicklung." In: 
DIN Deutsches Institut für Normung, e.V. Fachsprachen. Terminologie, Struktur, Normung. Heft 4. Berlin \& Köln: Beuth Verlag GmbH, S. 11-18.

BECKER, Jochen. (2013) Marketing-Konzeption: Grundlagen des zielstrategischen und operativen Marketing-Managements.10., überarb. und erw. Aufl. München: Vahlen.

BEIER, Rudolf. (1980) Englische Fachsprache. Stuttgart, Berlin, Köln \& Mainz: Kohlhammer.

BIRKENMAIER, Willy \& Irene Mohl. (1991) Russisch als Fachsprache. Tübingen: Francke.

Bolten, Jürgen. (1991) „Fremdsprache Wirtschaftsdeutsch: Bestandsaufnahme und Perspektiven." In: Müller, Bernd-Dietrich (Hrsg.) 1991. Interkulturelle Wirtschaftskommunikation. München: Iudicium Verlag, S. 71-91.

BolteN, Jürgen. (1998) „Fachsprachliche Phänomene in der Interkulturellen Wirtschaftskommunikation." In: Hoffmann, Lothar et al. (Hrsg.) 1998. Fachsprache. Ein internationales Handbuch zur Fachsprachenforschung und Terminologiewissenschaft. 1.Halbband. Berlin: Walter de Gruyter, S. 849-855.

BOLTEN, Jürgen. (2006) „Interkulturelle (Wirtschafts-) Kommunikation: „Fach“ oder "Gegenstandsbereich“? Wissenschaftshistorische Entwicklungen und studienorganisatorische Perspektiven.“ In: Moosmüller, Alois (Hrsg.) 2006. Interkulturelle Kommunikation. Konturen einer wissenschaftlichen Disziplin. Münster: Waxmann, S. 171-208.

BRUHn, Manfred. (2014) Marketing: Grundlagen für Studium und Praxis. 12., überarb. Aufl. Wiesbaden: Springer Gabler.

BRÜNNER, Gisela. (2000) Wirtschaftskommunikation. Linguistische Analyse ihrer mündlichen Formen. Tübingen: Max Niemeyer Verlag.

BudiN, Gerhard. (2002) „Wissensmanagement in der Translation.“ In: Best, Joanna \& Sylvia Kalina (Hrsg.) 2002. Übersetzen und Dolmetschen. Tübingen \& Basel: Francke, S. 74-84.

BungarTen, Theo. (1996) „Mehrsprachigkeit in der Wirtschaft.“ In: Goebl, Hans et al. (Hrsg.) 1996. Kontaktlinguistik. Ein internationales Handbuch zeitgenössischer Forschung.1. Halbband. Berlin: Walter de Gruyter, S. 414-421.

CABRÉ, María Teresa. (1999) La terminología: representación y comunicación: elementos para una teoría de base comunicativa y otros artículos. Textos en castellá, catalá i frances. Barcelona: Universidad Pompeu Fabra, Institut Universitari de Lingüística Aplicada II.

DONALIES, Elke. (2007) Basiswissen. Deutsche Wortbildung. Tübingen \& Basel: Francke Verlag.

EICHINGER, Ludwig M. (2000) Deutsche Wortbildung: eine Einführung. Tübingen: Narr. 
FLUCK, Hans-Rüdiger. (1984) Fachdeutsch in Naturwissenschaft und Technik. Einführung in die Fachsprachen und Didaktik/Methodik des fachorientierten Fremdsprachenunterrichts. Heidelberg: Groos.

FLUCK, Hans-Rüdiger. (1985) Fachsprachen. Einführung und Bibliographie. 3. aktualisierte u. erweiterte Auflage. Tübingen: Francke Verlag.

GAllego HeRnÁNDEZ, Daniel. (2012) Traducción económica y corpus: del concepto a la concordancia. Aplicación al francés y al español. Alicante: Publicaciones Universidad de Alicante.

GERZYMISCH-ARBOGAST, Heidrun. (1987) Zur Thema-Rhema-Gliederung in amerikanischen Wirtschaftsfachtexten. Eine exemplarische Analyse. Tübingen: Narr.

Gómez CAPuz, Juan. (2004) Préstamos del español: lengua y sociedad. Cuadernos de Lengua Española. Madrid: Arco Libros, S.L.

HAHN, Walter von. (1983) Fachkommunikation: Entwicklung, linguistische Konzepte, betriebliche Beispiele. Berlin \& New York: de Gruyter.

HelBIG, Gerhard \& Joachim Buscha. (2001) Deutsche Grammatik. Ein Handbuch für den Ausländerunterricht.19. Auflage. Berlin \& München: Langenscheidt KG.

HofFmann, Lothar. (1982) „Probleme und Methode der Fachsprachenforschung." In: Rodriguez Richart, José (Hrsg.) 1982. Fachsprachenforschung und -lehre: Schwerpunkt Span.; internat. Kolloquium an d. Univ. d. Saarlandes, Saarbrücken, 6-8. November 1980. Tübingen: Narr, S. 1-13.

Hoffmann, Lothar (Hrsg.) (1987) Fachsprachen, Instrument und Objekt. 1. Aufl. Leipzig: Verlag Enzyklopädie.

HofFmAnN, Lothar. (1993) „Fachwissen und Fachkommunikation. Zur Dialektik von Systematik und Linearität in den Fachsprachen." In: Bungarten, Theo (Hrsg.) 1993. Fachsprachentheorie. Bd. 2: Konzeptionen und theoretische Richtungen. Tostedt: Attikon, S. 595-617.

HoffmanN, Lothar. (1987) „Fachsprachen - Instrument und Objekt.“ In: Hoffmann, Lothar (Hrsg.) 1987. Fachsprachen, Instrument und Objekt. 1. Aufl. Leipzig: Verlag Enzyklopädie, S. 7-9.

HomburG, Christian. (2012) Grundlagen des Marketingmanagements. Einführung in Strategie, Instrumente, Umsetzung und Unternehmensführung. 3., überarbeitete und erweiterte Auflage. Wiesbaden: Springler Gabler.

HundT, Markus. (1995) Modellbildung in der Wirtschaftssprache: zur Geschichte der Institutionen- und Theoriefachsprachen der Wirtschaft. Tübingen: Niemeyer.

HundT, Markus. (1998) Neuere institutionelle und wissenschaftliche Wirtschaftsfachsprachen. In: Steger, Hugo \& Herbert Ernst Wiegand (Hrsg.) 1998. Handbücher zur Sprach- und Kommunikationswissenschaft. Bd. 14 Fachsprachen. Halbbd. 1. Berlin \& New York: de Gruyter, S. 1296-1304.

KlaCKLAUER, Alexander; D. Quinn Mills; Olaf Passenheim \& Dirk Seifert. (2001) „Online-Marketing-Controlling - Neue Wege in der Erfolgsmessung von 
Marketingmaßnahmen. " Der Markt - International Journal of Marketing 40:4, S. 151-158.

Kuss, Alfred. (2013) Marketing-Theorie: Eine Einführung. 3., überarb. u. erw. Aufl. Wiesbaden: Springer Gabler.

MEFFERT, Heribert; Christoph Burmann \& Manfred Kirchgeorg. (2015) Marketing: Grundlagen marktorientierter Unternehmensführung Konzepte - Instrumente Praxisbeispiele.12., überarb. u. aktualisierte Aufl. Wiesbaden: Springer Gabler. MÖHN, Dieter. (1980) „Zum Fortgang der germanistischen Fachsprachenforschung in den 70er Jahren." Zeitschrift für Germanistische Linguistik. 3, S. 352-369.

MÖHN, Dieter \& Roland Pelka. (1984) Fachsprachen: e. Einf. Tübingen: Niemeyer. MotsCH, Wolfgang. (2004) Deutsche Wortbildung in Grundzügen. 2., überarbeitete Auflage. Berlin \& New York: Walter der Gruyter.

MÜLLER, Bernd-Dietrich (Hrsg.) (1991) Interkulturelle Wirtschaftskommunikation. München: Iudicium-Verlag.

PÉREZ BERENGUEL, José Francisco. (2003) "Glosario de errores comunes en la traducción económica y financiera.” In: Muñoz Martín, Ricardo (Hrsg.) I AIETI. Actas del I Congreso Internacional de la Asociación Ibérica de Estudios de Traducción e Interpretación. Granada 12-14 de Febrero de 2003. Granada: AIETI. Vol. n. ${ }^{\circ}$ 2, pp. 619-628.

RoElCKE, Thorsten. (2010) Fachsprachen. 3., neu bearb. Aufl. Berlin: Schmidt.

SERÓN ORdóÑEZ, Inmaculada. (2005) "La traducción de la metáfora en los textos financieros: estudio de caso." In: Torres, María Gracia \& Marie-Ange Bugnot (Hrsg.) 2005. Traducción y cultura: el referente cultural en la comunicación especializada. Málaga: Libros ENCASA, pp. 205-250.

SCHMITT, Peter A. (1985) Anglizismen in den Fachsprachen: eine pragmatische Studie am Beispiel der Kerntechnik. Heidelberg: Winter.

Schmit, Peter A. (1990) Die Berufspraxis der Übersetzer. Eine Umfrageanalyse. Berichtssonderheft des Bundesverbandes der Dolmetscher und Übersetzer. Bonn: BDÜ.

STEGER, Hugo \& Herbert Ernst Wiegand (Hrsg.) (1998) Handbücher zur Sprachund Kommunikationswissenschaft. Bd. 14 Fachsprachen. Halbbd. 1. Berlin \& New York: de Gruyter.

WALSH, Gianfranco; Alexander Klee \& Thomas Kilian. (2009) Marketing: eine Einführung auf der Grundlage von Case Studies. Berlin \& Heidelberg: Springer.

WÖHE, Günter. (2012) Einführung in die Allgemeine Betriebswirtschaftslehre. 25. Auflage. München: Verlag Franz Vahlen. 
Wörterbücher:

Alcaráz VAro, Enrique. (2008) Diccionario de términos económicos, financieros y comerciales. Inglés - Español, Spanish - English. 5., überarb. und erw. Aufl. Barcelona: Ariel.

JÖRCKEL, Sabine. (1998) Wirtschaftsspanisch: Einführung. München \& Wien: Oldenbourg.

PAdilla GálVEZ, Jesus. (1998) Wirtschaftsspanisch: Marketing: Estudio de casos de marketing. München \& Wien: Oldenbourg.

SÁNCHEZ, Celestino (2007) Fachwörterbuch. Wirtschaft, Handel und Finanzen. Spanisch-Deutsch, Deutsch-Spanisch. 1. Auflage. Berlin u.a.: Langenscheidt.

SCHNitZER, Johannes \& Jordi Martí (Hrsg.) (1996) Wirtschaftsspanisch: terminologisches Handbuch = Manual de lenguaje económico. München: Oldenbourg.

Online-Quellen:

www.duden.de

www.rae.es

www.puromarketing.com

www.merca20.com

www.liderazgoymercadeo.com 


\section{KURZBIOGRAPHIE / BIONOTE}

Guadalupe Ruiz YePes ist Professorin für Spanisch als Fachsprache für Wirtschaft und regionale Studien der hispanophonen Länder an der Hochschule Heilbronn. Sie promovierte im Bereich Übersetzungswissenschaft und beschäftigt sich derzeit mit der kontrastiven Analyse der Wirtschaftsphraseologie. Im Rahmen eines Forschungsaufenthalts als Post-Doc hat sie ein Jahr an der Aston University verbracht. Sie hat Übersetzung und Sprachen an Universitäten in Spanien, Deutschland, Großbritannien und den USA gelehrt.

GuAdAluPe Ruiz YePES is a professor of Business Spanish and Cultural Studies of the Hispanic World at Heilbronn University. She holds a Ph.D. in Translation Studies. Her research interests are currently focusing on contrastive analysis of business phraseology. She spent a year at Aston University as a post-doctoral research fellow and has also taught translation and foreign languages in higher education in the USA and Spain.

ANASTASIA KonOvalova hat im Jahre 2005 den Studiengang für Wirtschaft und im Jahre 2007 den Studiengang für Linguistik und Fremdsprachen (Spanisch und Deutsch) absolviert. 2009 wurde sie im Bereich Wirtschaftswissenschaften promoviert. Sie ist Dozentin für Wirtschaftsspanisch an der Hochschule Heilbronn. Seit 2010 ist sie Doktorandin am Institut für Übersetzen und Dolmetschen (Universität Heidelberg). Sie beschäftigt sich mit der vergleichenden Analyse der Wirtschaftsterminologie im Deutschen, Spanischen und Russischen.

ANASTASIA KONOvalova graduated in Economics in 2005 and in Linguistics and Foreign Languages (Spanish and German) in 2007. She earned a Ph.D. in Economics in 2009. She teaches Business Spanish at Heilbronn University. Since 2010, she has been a post-graduate student at the Institute for Translation and Interpretation (University of Heidelberg). Her research interests include the comparative analysis of economic terminology in German, Spanish and Russian. 